\title{
Characteristics of myosin isoforms in mammalian skeletal muscle
}

\author{
Priit Kaasik $^{1}$, Kätlin Leisson ${ }^{2}$, Raivo Puhke ${ }^{1}$, Karin Alev $^{1}$, Teet Seene ${ }^{{ }^{*}}$ \\ ${ }^{1}$ Institute of Exercise Biology and Physiotherapy, University of Tartu, Tartu, Estonia \\ ${ }^{2}$ Institute of Veterinary Medicine and Animal Sciences, Estonian University of Life Sciences, Tartu, Estonia \\ Email: "teet.seene@ut.ee
}

Received 21 February 2012; revised 13 April 2012; accepted 18 April 2012

\begin{abstract}
The distribution of myosin heavy (MyHC) and myosin light chain (MyLC) isoform pattern in horse, rat and human skeletal muscle was investigated to establish relations between them and the role of myosin isoform patterns in mammalian muscle with different twitch characteristics was studied. These two isoforms were separated in a SDS-PAGE gel system, stained using the coomassie and silver staining procedures, and the results were analyzed using a G:BOX system. The relative content of MyHC I isoform in muscle was 2.6 times higher than in human compared to horse muscle $(p<0.001)$, and 6.3 times higher than in rat muscle $(p<0.001)$. The relative content of MyHC IIx/d isoform in horse muscle is 2.7 times, and in rat muscle 2.2 times higher in comparison with human muscle $(p<0.001)$. The role of the MyLC isoform distribution in mammalian skeletal muscle seems to depend on the oxidative capacity of muscles.
\end{abstract}

Keywords: Myosin Isoforms; Skeletal Muscle; Horses; Rats and Humans

\section{INTRODUCTION}

Myosin controls the functional properties of skeletal muscle fibers. Nearly fifty years ago, Barany showed that maximal shortening velocity within skeletal muscle of various mammals was related to actomyosin ATPase activity [1], and the major component of myosin molecule the MyHC isoform was responsible for this $[2,3]$. Myosin exists in striated muscle as multiple isoforms due to the polymorphic expression of both its heavy and light chain components [4]. The molecule is found in two $\mathrm{MyHC}$ and four MyLC isoforms, three essential MyLC isoforms (MyLC $1_{\text {fast }}$, MyLC $1_{\text {slow }}$, and MyLC $3_{\text {fast }}$ ) and two regulatory MyLC isoforms (MyLC 2 fast and MyLC

\footnotetext{
"Corresponding author.
}

$2_{\text {slow }}$ ). The role of essential MyLC, particularly MyLC $3_{\text {fast }}$, is related to the shortening velocity in single fast-twitch (FT) fibers [5]; additionally, it has a modulatory role in the regulation of cross-bridge kinetics [6-9]. Maximal shortening velocity in single fibers expressing the same MyHC isoform increases as the MyLC 3 fast MyLC $1_{\text {fast }}$ ratio increases. The electrophoretic mobility of MyLC $1_{\text {fast }}$ varies among mammalian species with different body mass [10]. Differences in MyLC fast isoform molecular mass between species are related to differences in amino acid sequence, the length of the common polyalanine region near the $\mathrm{NH}_{2}$-terminal actinbinding site [11]. The MyLC $1_{\text {fast }}$ isoform relative content decreases in rat skeletal muscles in the following directions: extensor digitorum longus-plantaris-diaphragmsoleus [12]. The statement by Hayashibara and Miyanishi [13] that MyLC $1_{\text {fast }}$ isoform's relative content in muscle indicates the rate of slowdown of muscle contraction has a functional outcome. However, it has been proposed that the functional role of MyLC isoforms is realized in combination with certain $\mathrm{MyHC}$ isoforms as higher affinities of certain MyLC and MyHC isoforms exist $[14,15]$, e.g. the higher affinity between MyLC $3_{\text {fast }}$ isoform and MyHC IIb and IId isoforms. A wide distribution of predominant slow isoforms in slow-twitch (ST) soleus muscle of rats show the physiological role and adaptability of MyLC isoforms to everyday motor activity as these muscles participate in the process of slow movements and keeping static positions [12]. Changes in skeletal muscle MyHC isoform composition with advancing age have also been explained by differences in the distribution of mechanical loading of muscles between bipedals and quadripedals [16]. MyHC fastest isoform IIb isoform has been shown to be species-specific [17] and muscles IIb isoform has only been shown to be expressed in rodents [18], marsupials [19] and as an exception among large animals in the pig hybrid IIx/b fibers [20]. As external factors, such as functional activity or inactivity, may influence myosin isoform composition $[21,22]$, we used sedentary humans, horses, and rats 
in the present study.

The MyLC and MyHC isoforms distribution and their physiological role in the skeletal muscle of large and small sized quadripedals and between muscles with different twitch characteristics in comparison with human muscle is so far unclear. Clarification of the abovementioned problems is significant in understanding the biological and physiological peculiarities of muscle contractile properties of small and large sized quadripedals and humans.

This study investigated the distribution of $\mathrm{MyHC}$ and MyLC isoforms pattern in horse, rat, and human skeletal muscle to clarify the role of myosin isoforms in skeletal muscles of small and large mammalian and human muscle as well as in muscle with differences in twitch characteristics.

\section{MATERIALS AND METHODS}

\subsection{Declaration}

10 sedentary male humans (body mass $79.6 \pm 2.2 \mathrm{~kg}$, $40.0 \pm 2.1$ years old), 10 Akhal-Teke horses (body mass $468.0 \pm 8.6 \mathrm{~kg}, 14.0 \pm 1.2$ years old) and 12 Wistar rats (body mass $345.0 \pm 6.2 \mathrm{~g}, 22$ weeks old) were included in this study.

The methods and procedures used on all the animals in this study were performed in accordance with the European Communities Directive (86/609/EEC) and permit (No 9, September 5, 2006) from the Estonian National Board of Animal Experiments. All subjects voluntarily gave an informed, written consent and the study was undertaken in accordance with the Declaration of Helsinki [23]. All subjects, whose samples were obtained for this study, were healthy. Authors have no conflict of interest.

\subsection{Muscle Biopsy and Tissue Preparation Procedures}

Humans. Muscle biopsies were taken from middle-aged sedentary men under local anesthesia from the left Vastus lateralis muscle using the percutaneous conchotome technique.

Horses. Muscle biopsies were taken at a depth of 60 $\mathrm{mm}$ from the left Gluteus medius muscle approximately $15 \mathrm{~cm}$ caudodorsal to the tuber coxal using a needle - the biopsy technique by Ledwith and McGowan [24]. The muscle biopsies were frozen in liquid nitrogen.

Rats were anaesthetized by an intraperitoneal injection of ketamin (Calysol, Gedeon Richter A.O. Budapest, Hungary) and diazepam (Lab Renaudin, France). The plantaris (Pla), extensor digtorum longus (EDL), soleus (Sol), and diaphragm (Dia) muscles were quickly removed, trimmed clean of visible fat and connective tis- sue, weighed, and frozen in liquid nitrogen. All collected muscle samples were preserved at $-80^{\circ} \mathrm{C}$ until further analysis.

\subsection{Myofibrillar Protein Extraction and Sample Preparation}

A portion $(30 \mathrm{mg})$ of each muscle was crushed to a fine powder in liquid nitrogen using mortar and pestle. The powdered samples were homogenized in ice-cold wash buffer $\left(20 \mathrm{~mm} \mathrm{NaCl}, 5 \mathrm{mM} \mathrm{Na}_{2} \mathrm{HPO}_{4}, 1 \mathrm{mM}\right.$ EGTA pH $=6.5$ ). The homogenate was centrifuged at $10,000 \mathrm{rpm}$ for $10 \mathrm{~min}$ at $4^{\circ} \mathrm{C}$. The pellet was suspended in extraction buffer $\left(100 \mathrm{mM} \mathrm{Na} \mathrm{P}_{2} \mathrm{O}_{7}, 5 \mathrm{mM}\right.$ EGTA, $1 \mathrm{mM}$ dithiotreitol $\mathrm{pH}=8.5$ ) for 1 hour at $4^{\circ} \mathrm{C}$. The samples were then centrifuged at $10,000 \mathrm{rpm}$ for 10 minutes at $4^{\circ} \mathrm{C}$. Supernatant was collected and mixed with an equal volume of glycerol and stored at $-80^{\circ} \mathrm{C}$ [25].

The protein concentration was determined using a colorimetric assay (Bio-Rad, Hercules, CA) and standardized with sample buffer $(62.5 \mathrm{mM}$ Tris $(\mathrm{pH}=6.8)$, $5 \% \beta$-mercaptoethanol, $2.5 \%$ SDS, $20 \%$ glycerol, and $0.1 \%$ bromphenol blue) to $0.1 \mathrm{mg} / \mathrm{ml}$ (MyHC) and 1 $\mathrm{mg} / \mathrm{ml}$ (MyLC). Samples were heated for 3 minutes at $100^{\circ} \mathrm{C}$, immediately chilled on ice, and stored in a $-80^{\circ} \mathrm{C}$ freezer until they were run on SDS gels.

\subsection{MyHC and MyLC Isoforms Determination}

$\mathrm{MyHC}$ isoforms from human and rat muscle samples were separated by the previously described method [26] with the separating gel containing $7.2 \%$ acrylamide, 372 $\mathrm{mM}$ Tris- $\mathrm{HCl}(\mathrm{pH}=8.6), 30 \%$ glycerol, $0.1 \%$ SDS, and the stacking gel containing $4 \%$ acrylamide, $125 \mathrm{mM}$ Tris- $\mathrm{HCl}(\mathrm{pH}=6.7)$, and $0.1 \%$ SDS. The running buffer contained $25 \mathrm{mM}$ Tris, $192 \mathrm{mM}$ glycine, $0.1 \%$ SDS $(\mathrm{w} / \mathrm{v})$.

Electrophoresis was run for $24 \mathrm{hrs}$ at a low temperature with $120 \mathrm{~V}$ (constant voltage).

The gels used to examine MyHC isoform composition of horse muscle samples were prepared as previously described by Talmadge and Roy [27].

The separating gel contained $8 \%$ acrylamide, $200 \mathrm{mM}$ Tris- $\mathrm{HCl}(\mathrm{pH}=8.8), 100 \mathrm{mM}$ glycine, $30 \%$ glycerol and $0.4 \%$ SDS, whereas the stacking gel contained $4 \%$ acrylamide, $70 \mathrm{mM}$ Tris- $\mathrm{HCl}(\mathrm{pH}=6.8), 4 \mathrm{mM}$ EDTA, $30 \%$ glycerol and $0.4 \%$ SDS.

The lower running buffer contained $50 \mathrm{mM}$ Tris, 75 $\mathrm{mM}$ glycine and $0.05 \%$ SDS, while the top running buffer contained twice the abovementioned concentrations. This protocol was slightly modified to improve the separation of MyHC isoforms by addition of reducing agent $\beta$-mercaptoethanol (final concentration: $0.12 \%$ ) to the top running buffer [28]. Electrophoresis was run for $20 \mathrm{hrs}$ at a low temperature with $140 \mathrm{~V}$ (constant volt- 
age).

All MyHC gels were fixed and silver-stained by the method of Oakley et al. [29].

MyHC isoforms (MyHC I, MyHC IIa, MyHC IIx/d, MyHC IIb) (Figure 1(a)) were quantified densitometrically by a computer-based image analysis system and software (Image Master 1D, Version 4.0, Amersham Pharmacia Biotech, Newcastle upon Tyne, England).

The MyLC isoforms (from human, horse, and rat muscle samples) were separated by $12.5 \%$ SDS-PAGE gel system according to Laemmli [30], except that the glycerol content in the separating gel was $10 \%$. Electrophoresis was performed using the vertical slab gel system at a constant current $(10 \mathrm{~mA})$ and stopped when the dye front reached the bottom of the gels. The gels were fixed and stained using the colloidal Coomassie staining procedure (Coomassie Brilliant Blue G-250 with phosphoric acid in the presence of ammonium sulfate) according to Neuhoff [31]. Following staining, the MyLC gels were scanned and band (MyLC $1_{\text {slow }}$, MyLC $1_{\text {fast }}$, MyLC $2_{\text {slow, MyLC } 2 \text { fast }}$ and MyLC $3_{\text {fast }}$ isoform) (Figure 1(b)) densities were analyzed using gel documentation and analysis system G:BOX (GeneSnap, Genetools, Syngene, UK).

\subsection{Statistical Analysis}

Data were managed and analyzed using software packages MS Office Excel 2003 and SAS 9.1. The one-way ANOVA was used to test the statistical significance of

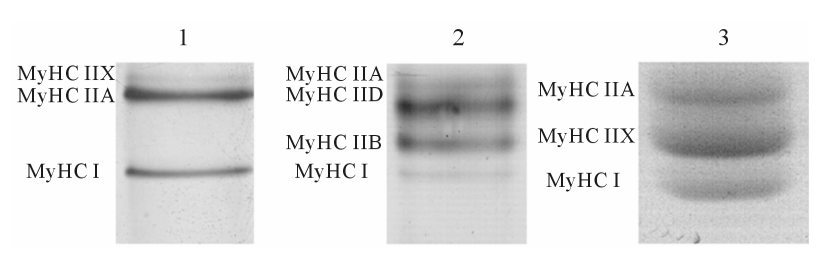

(a)

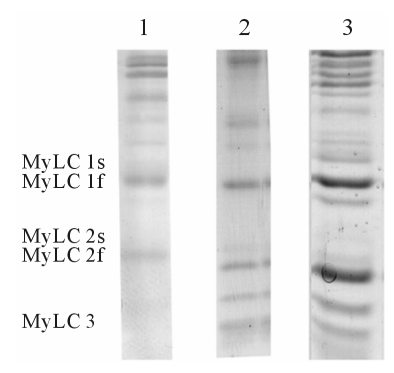

(b)

Figure 1. MyHC (a) and MyLC (b) isoform pattern in human Musculus vastus lateralis (1) rat Musculus plantaris (2) and horse Musculus glutus medius (3). MyHC isoforms from human and rat muscle samples were separated by the method of Hämäläinen and Pette [26] from horse muscle samples by the method Talmadge and Roy [27]. MyLC isoforms from human, rat and horse muscle samples were separated by the method of Laemmli [30]. differences between age groups, and Pearson correlation coefficients were calculated. Data are presented as mean \pm SE. Significance level was set at $\mathrm{P} \leq 0.05$.

\section{RESULTS AND DISCUSSION}

\subsection{MyHC and MyHC Isoforms in Human, Horse and Rat Skeletal Muscle}

Data obtained from human, horse and rat skeletal muscle show that there are significant differences in the distribution of MyHC isoforms (Figure 2(a)) and MyLC isoforms relative content (Figure 2(b)). MyHC I and IIa isoforms content is significantly higher in human muscle, although there are significant differences between horses and rats. Contrary to MyHC I and IIa relative content, $\mathrm{MyHC} \mathrm{IIx} / \mathrm{d}$ isoforms relative content is more than twice

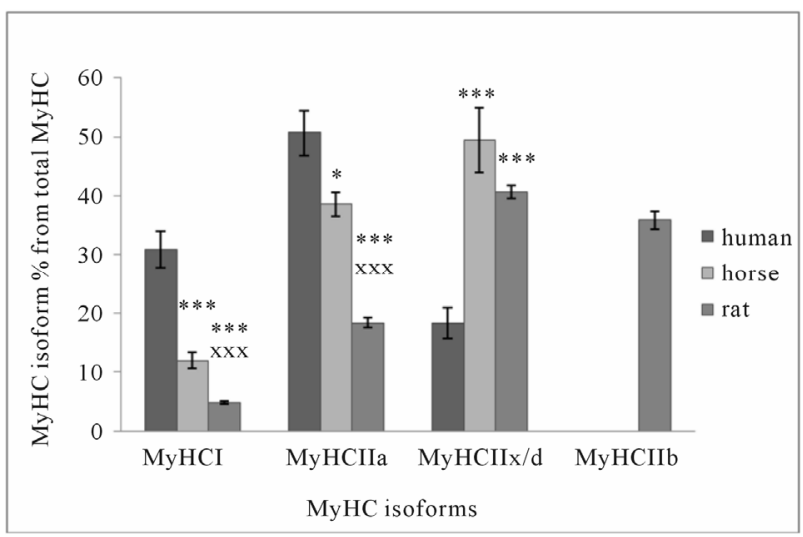

(a)

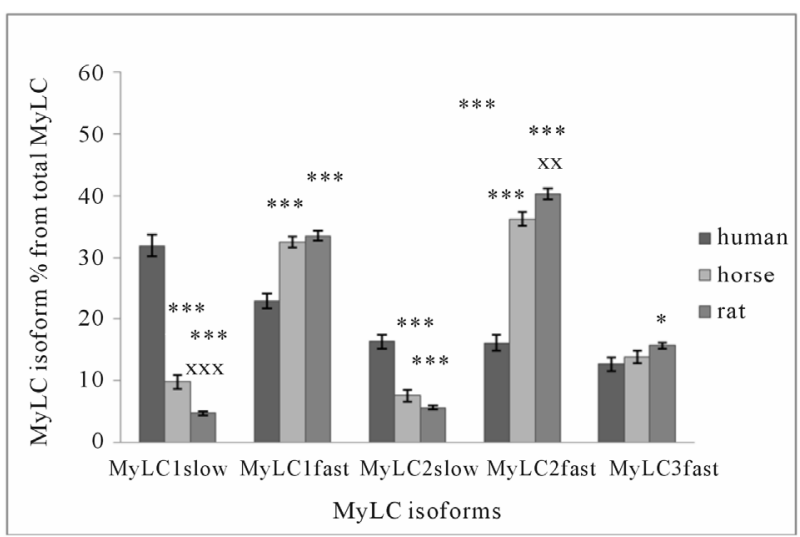

(b)

Figure 2. MyHC (a) and MyLC (b) isoform composition in human, horse, and rat skeletal muscle. Biopsy was taken from musculus Quadriceps femoris of 10 men (average age $40 \pm 6$ years, body mass $79.6 \pm 9.8 \mathrm{~kg}$ ), and from Musculus gluteus medius of 10 Akhal-Teke horses (average age $14 \pm 1.2$ years, body mass $468 \pm 8.6 \mathrm{~kg}$ ), and from Musculus plantaris of Wistar rats $\left(22\right.$ weeks old, body mass $345 \pm 6.2 \mathrm{~g}$ ). ${ }^{*} \mathrm{p}<0.05$ in comparison with human; ${ }^{* * *} \mathrm{p}<0.001$ in comparison with human; ${ }^{x x} p<0.01$ in comparison with horse; ${ }^{x x x} p<0.001$ in comparison with horse. 
higher in horse and rat muscle (Figure 2(a)). The sum of MyHC I and IIa isoforms is about $82 \%$ of total MyHC in human, $51 \%$ in horse, and $23 \%$ in rat muscle. These isoforms are characteristic to specific muscle fibers with high oxidative capacity. The sum of all fast MyHC isoforms $(\mathrm{IIa}+\mathrm{IIx} / \mathrm{d}+\mathrm{IIb})$ is about $95 \%$ in rat, $88 \%$ in horse, and $69 \%$ in human skeletal muscle. The sum of the fastest MyHC isoforms (IIx/d + IIb), which are characteristic to specific fast-twitch fast fatigue muscle fibers, is about $77 \%$ in rat, $49 \%$ in horse, and $18 \%$ in human muscle. Figures 2(a), 3(a), and 3(b) show that in muscles where MyHC I isoforms relative content is higher, there is a higher percentage of MyLC slow isoforms and vice versa. In human skeletal muscle, the sum of MyLC slow isoforms is about $48 \%$, in horse $17 \%$ and in rat $10 \%$ (Figure 2(b)). A comparison of the same sum in different rat muscles shows that in well-known ST muscle Sol, it

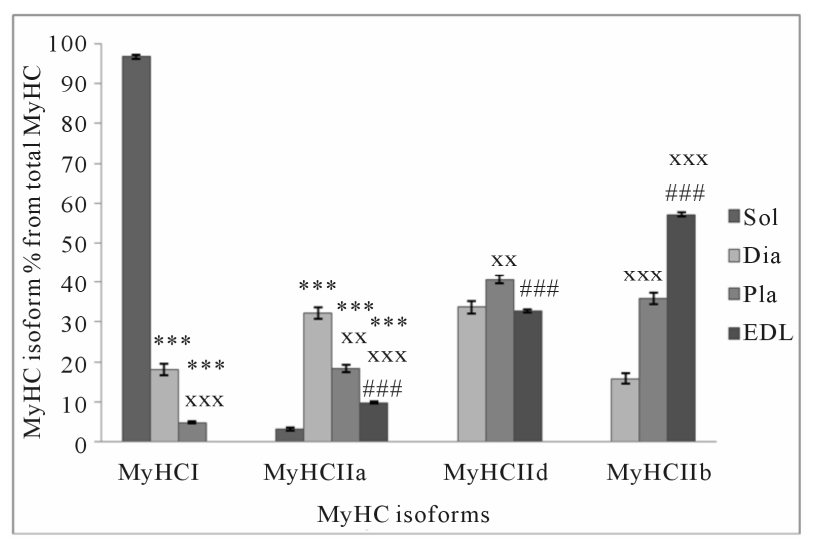

(a)

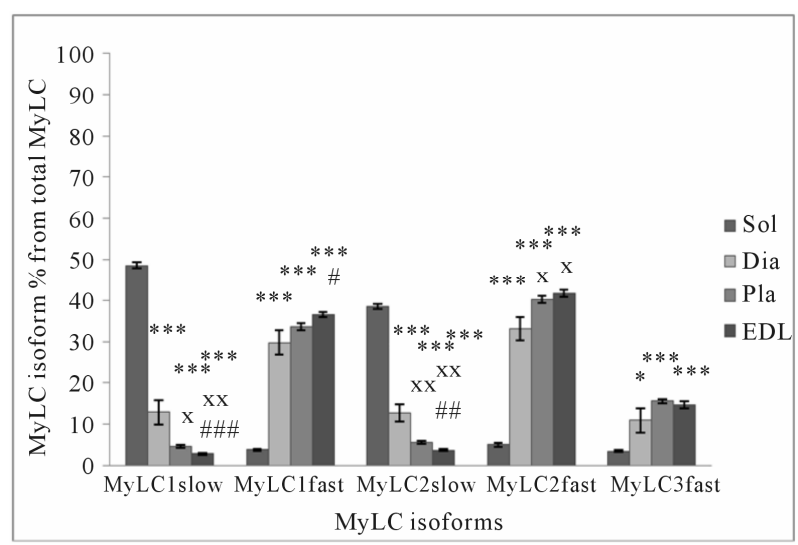

(b)

Figure 3. MyHC (a) and MyLC (b) isoform composition in rat slow-twitch and fast-twitch muscles. ${ }^{*} \mathrm{p}<0.05$ in comparison with soleus muscle; ${ }^{* *} \mathrm{p}<0.01$ in comparison with soleus muscle; ${ }^{* * *} \mathrm{p}<0.001$ in comparison with soleus muscle; ${ }^{\mathrm{x}} \mathrm{p}<0.05$ in comparison with diaphragm; ${ }^{\mathrm{xx}} \mathrm{p}<0.01$ in comparison with diaphragm; ${ }^{\mathrm{xx}} \mathrm{p}<0.001$ in comparison with diaphragm; ${ }^{*} \mathrm{p}<$ 0.05 in comparison with plantaris muscle; ${ }^{\#} \mathrm{p}<0.01$ in comparison with plantaris muscle; ${ }^{\# \#} \mathrm{p}<0.001$ in comparison with plantaris muscle. is about $87 \%$ and in FT muscle EDL, it is about $7 \%$ (Figure 3(b)). The sum of MyLC fast isoforms is about 52\% in human, $83 \%$ in horse, and $89 \%$ in rat muscle (Figure 2(b)). In rat ST Sol muscle, this sum is about $13 \%$, and FT EDL muscle about 93\%. If the sum of MyLC 1 and 3 isoforms (essential isoforms) is about $55 \%$ in horse and rat muscle, and the sum of MyLC 2 isoforms (regulatory isoforms) is about $45 \%$, the sum of essential MyLC isoforms in human muscle is significantly higher (about $68 \%, \mathrm{p}<0.05)$ and the sum of regulatory MyLC isoforms is significantly lower (about $32 \%, \mathrm{p}<0.05$ ) in comparison with horse and rat muscle. The main difference between MyLC isoforms' relative content in human, horse and rat skeletal muscle is the low level of regulatory MyLC content in human muscle. MyLC 3 fast $/$ MyLC $1_{\text {fast }}$ ratio in human muscle is $0.55 \pm 0.04$, in horse $0.43 \pm$ $0.02(\mathrm{p}<0.01)$, and in rat muscle $0.47 \pm 0.001$.

\subsection{Correlation between MyHC and MyLC Isoforms Relative Content}

There was a positive correlation between MyHC I isoform and MyLC $1_{\text {slow }}$ and $2_{\text {slow }}$ isoforms relative content $(\mathrm{r}=0.73$, and 0.71 respectively; $\mathrm{p}<0.01)$ and $\mathrm{MyHC} \mathrm{IIb}$ isoform and MyLC $1_{\text {fast }}, 2_{\text {fast }}$ and $3_{\text {fast }}$ isoforms $(\mathrm{r}=0.74$, 0.72 , and 0.73 respectively; $p<0.01$ ) in rat muscles with different twitch characteristics. In human and horse muscle, a positive correlation was found between MyHC IIx isoform relative content and MyLC $1_{\text {fast }}, 2_{\text {fast }}$ isoforms ( $\mathrm{r}$ $=0.72,0.70$, and 0.75 respectively).

\subsection{Role of MyHC and MyLC Isoforms in Muscle Function}

Among multiple isoforms of muscle proteins, $\mathrm{MyHC}$ and MyLC isoforms play an important role in muscle function. If the role of $\mathrm{MyHC}$ isoforms in skeletal muscle function regulation of the contractile speed is wellknown [32,33], the role of MyLC isoforms in contractile machinery has not been fully determined. The number of MyHC isoforms expressed in skeletal muscle depends on the species. In large mammals, three isoforms are expressed in skeletal muscle (MyHC I, MyHC IIa, and MyHC IIx) [16,34]. Proof of the abovementioned standpoint about the functional role of myosin isoforms is shown in the studies, where MyLC $1_{\text {fast }}$ and MyLC $2_{\text {fast }}$ isoforms relative content was found to increase in parallel with the increase in relative content of MyHC IIb isoform during muscle atrophy [4]. It was shown that the decrease in the relative content of MyLC $1_{\text {fast }}$ isoform is an indicator of slowing of muscle contraction $[12,13]$. The present study shows that there is a positive correlation between the relative content of MyHC I isoform and MyLC $1_{\text {slow }}$ and MyLC $2_{\text {slow }}$ isoforms and a negative correlation between MyLC $1_{\text {fast }}$, MyLC $2_{\text {fast, }}$, and MyLC 
$3_{\text {fast }}$ isoforms relative content. A positive correlation between MyHC IIx/d isoforms and MyLC $1_{\text {fast, }}, 2_{\text {fast }}$, and $3_{\text {fast }}$ isoforms relative content in skeletal muscle was found. The highest percent of relative content of MyHC I isoform and MyHC I and MyHC IIa isoform sum was found in human skeletal muscle. In horse muscle, the sum of MyHC I and IIa isoforms was about 38\% less, and in rat muscle about $72 \%$ less in comparison with human muscle. As MyHC I isoform relative content in human muscle was 2.6 times higher than in horse and 6.3 times higher than in rat muscle, it may show the differences in endurance capacity of human, horse, and rat muscle.

\subsection{Role of Muscle Oxidative Capacity in Regulation of MyHC and MyLC Isoforms Relative Content}

The abovementioned differences between human, horse, and rat muscle MyHC I and IIa isoforms sum are characteristic to the function of the contractile machinery in muscles with high oxidative capacity as a high relative content of MyHC I and IIa isoforms is characteristic to muscle fibers with high oxidative capacity $[31,35]$. The relation between the relative content of MyHC fastest isoform and MyLC $3_{\text {fast }}$ isoform and muscle contractile speed has been shown to exist [36]. The present study did not show a correlation between MyLC $3_{\text {fast }} /$ MyLC $1_{\text {fast }}$ isoforms ratio and the fastest MyHC isoform relative content. The positive correlation between MyHC IIx/d isoform relative content and both essential and regulatory MyLC fast isoforms relative content support the opinion that MyLC $1_{\text {fast }}$ isoform relative content is an indicator of muscle contraction speed as has previously been shown $[12,13]$.

The main difference between the distribution of MyLC isoforms in human, horse, and rat skeletal muscle is the relatively low level of regulatory MyLC isoforms in human skeletal muscle. It has been shown earlier that muscle oxidative capacity plays an important role in the regulation of MyHC and MyLC isoforms content [12, 35]. MyLC isoforms distribution in skeletal muscle has been shown to be related to the physiological role and adaptational capacity of muscle to everyday motor activity [12]. The wide distribution of MyLC slow isoforms in ST rat Sol muscle shows the adaptational peculiarities of the muscle via these isoforms [12]. In the present study, we found that in spite of differences in MyHC isoforms pattern between quadripedals horse and rat skeletal muscle, in human muscle the relative content of MyHC I isoform is 2.6 times higher than in horse muscle and 6.3 times higher than in rat muscle. A comparison of the distribution of myosin isoforms pattern in human Vastus lateralis muscle and horse Gluteus medius muscle may raise a question about the comparability of MyHC I isoform relative content in these two muscles. Our previous study has shown that in human Gluteus medius muscle, MyHC I isoform content is about 63\% [37]. Thus, the MyHC I isoform relative content in both human skeletal muscles studied is much higher in comparison with horse and rat muscle. It may also be that variations in $\mathrm{MyHC}$ isoforms are not sufficient for fulfilling motor functions of muscles, and other myofibrillar proteins or posttranslational modifications of these proteins may have a significant role in the regulation of main muscle function $[38,39]$. Although among horses, the Akhal-Teke breed is known for great endurance and speed capacity [34], the relative content of MyHC IIx/d isoform is about 2.7 times higher in horse muscle and about 2.2 times higher in rat muscle than in human muscle. There is also a higher relative content of MyLC $1_{\text {fast }}$ and MyLC $2_{\text {fast }}$ isoforms in horse and rat muscle. Further investigation is needed to determine if these results are sufficient to state that there are differences between bipedals and quadripedals in $\mathrm{MyHC}$ and $\mathrm{MyLC}$ isoform distribution in skeletal muscle. MyHC IIb isoform relative content is about $36 \%$ in rat Pla muscle. MyHC IIb isoform is absent in skeletal muscle of horses, whose body mass is about 1400 times higher than that of rats.

\section{CONCLUSION}

In summary, the comparison of MyHC and MyLC isoforms pattern in human quadriceps femoris muscle, Akhal-Teke horse Gluteus medius muscle, and Wistar rat Pla muscle show that MyHC I isoform relative content in human muscle is 2.6 times higher than in horse muscle and 6.3 times higher than in rat muscle. The relative content of MyHC IIx/d isoform in horse muscle is about 2.7 times, and in rat muscle about 2.2 times higher than in human muscle. There is also a higher relative content of MyLC $1_{\text {fast }}$ and MyLC $2_{\text {fast }}$ isoforms in horse and rat muscle. The main difference in MyLC isoforms pattern between human, horse, and rat muscle is the low percent of regulatory MyLC isoforms (32.4\%) in human muscle, $44 \%$ and $46 \%$ respectively in horse and rat muscle. Quadripedals have a higher percent of MyHC IIx/d isoforms and MyLC $1_{\text {fast }}$ and MyLC $2_{\text {fast }}$ isoforms relative content in skeletal muscle than in bipedals human muscle. The role of MyLC isoforms distribution in mammalian skeletal muscle seems to be related to MyHC isoforms pattern and depends on the oxidative capacity of muscle. Quadripedals with high body mass and humans do not express MyHC IIb isoform in skeletal muscle.

\section{ACKNOWLEDGEMENTS}

This study was supported by the funds of the Ministry of Education and Research of the Republic of Estonia, research project number 
TKKSB1787.

We would like to thank Helen Kaptein for English language editing and Piret Pärsim for technical expertise.

\section{REFERENCES}

[1] Barany, M. (1967) ATPase activity of myosin correlated with speed of muscle shortening. The Journal of General Physiology, 50, 197-216. doi:10.1085/jgp.50.6.197

[2] Moss, R.L., Diffee, G.M. and Greaser, M.L. (1995) Contractile properties of skeletal muscle fibers in relation to myofibrillar protein isoforms. Reviews of Physiology, Biochemistry and Pharmacology, 126, 1-63. doi:10.1007/BFb0049775

[3] Schiaffino, S. and Reggiani, C. (1996) Molecular diversity of myofibrillar proteins: Gene regulation and functional significance. Physiological Reviews, 76, 371-423.

[4] Diffee, G.M., Haddad, F., Herrick, R.E. and Baldwin, K.M. (1991) Control of myosin heavy chain expression interaction of hypothyroidism and hindlimb suspension. American Journal of Physiology Cell Physiology, 261, C1099-C1106.

[5] Greaser, M.L., Moss, R.L. and Reiser, P.J. (1988) Variations in contractile properties of rabbit single muscle fibres in relation to troponin $\mathrm{T}$ isoforms and myosin light chains. The Journal of Physiology, 406, 85-98.

[6] Bottinelli, R., Betto, R., Schiaffino, S. and Reggiani, C. (1994) Unloaded shortening velocity and myosin heavy chain and alkali light chain isoform composition in rat skeletal muscle fibres. The Journal of Physiology, 478, 431-449.

[7] Lowey, S., Waller, G.S. and Trybus, K.M. (1993) Function of skeletal muscle myosin heavy and light chain isoforms by an in vitro motility assay. The Journal of Biological Chemistry, 268, 20414-20418.

[8] Lowey, S., Waller, G.S. and Trybus, K.M. (1993) Skeletal muscle myosin light chains are essential for physiological speeds of shortening. Nature, 365, 454-456. doi: $10.1038 / 365454 \mathrm{a} 0$

[9] Sweeney, H.L., Kushmerick, M.J., Mabuchi, K., Sréter, F.A. and Gergely, J. (1988) Myosin alkali light chain and heavy chain variations correlate with altered shortening velocity of isolated muscle fibers. The Journal of Biological Chemistry, 263, 9034-9039.

[10] Bicer, S. and Reiser, P.J. (2004) Myosin light chain isoform expression among single mammalian skeletal muscle fibers: Species variations. Journal of Muscle Research and Cell Motility, 25, 623-633. doi:10.1007/s10974-004-5070-9

[11] Bicer, S. and Reiser, P.J. (2007) Variations in apparent mass of mammalian fast-type myosin light chains correlate with species body size, from shrew to elephant. American Journal of Physiology Regulatory, Integrative and Comparative Physiology, 292, R527-R534. doi:10.1152/ajpregu.00098.2006

[12] Alev, K., Kaasik, P., Pehme, A., Aru, M., Parring, A.-M., Elart, A. and Seene, T. (2009) Physiological role of myosin light and heavy chain isoforms in fast- and slow-twitch muscles: Effect of exercise. Biology of Sport, 26, 215-
234.

[13] Hayashibara, T. and Miyanishi, T. (1994) Binding of the amino-terminal region of myosin alkali 1 light chain to actin and its effect on actin-myosin integration. Biochemistry, 33, 12821-12827.

[14] Stevens, L., Firinga, C., Gohlsch, B., Bastide, B., Mounier, Y. and Pette, D. (2000) Effects of unweighting and clenbuterol on myosin light and heavy chains in fast and slow muscle of rat. American Journal Physiology Cell Physiology, 279, C1558-C1563.

[15] Wada, M. and Pette, D. (1993) Relationships between alkali light-chain complement and myosin heavy-chain isoforms in single fast-twitch fibers of rat and rabbit. European Journal of Biochemistry, 214, 157-161. doi:10.1111/j.1432-1033.1993.tb17908.x

[16] Kim, J., Hinchcliff, K.W., Yamaguchi, M., Beard, L.A., Markert, C.D. and Devor, S.T. (2005) Exercise training increases oxidative capacity and attenuates exercise-induced ultrastructural damage in skeletal muscle aged horses. Journal of Applied Physiology, 98, 334-342.

[17] Mascarello, F., Patruno, M., Toniolo, L., Reggiani, C. and Maccatrozzo, L. (2009) Phenothypic expression of $2 b$ myosin heavy chain isoform: A comparative study among species and different muscles. Veterinary Research Communications, 33, 105-107. doi:10.1007/s11259-009-9301-9

[18] Lucas, C.A., Kang, L.H. and Hoh, J.F. (2000) Monospecific antibodies against the three mammalian fast limb myosin heavy chains. Biochemical and Biophysical Research Communications, 272, 303-308. doi:10.1006/bbrc.2000.2768

[19] Zhong, W.W., Lucas, C.A., Kang, L.H. and Hoh, J.F. (2001) Electrophoretic and immunochemical evidence showing that marsupial limb muscles express the same fast and slow myosin heavy chains as eutherians. Electrophoresis, 22, 1016-1020.

doi:10.1002/1522-2683()22:6<1016::AID-ELPS1016>3.0 .CO;2-K

[20] Toniolo, L., Patruno, M., Maccatrozzo, L., Pellegrino, M., Canepari, M., Rossi, R., D’Antona, G., Bottinelli, R., Reggiani, C. and Mascarello, F. (2004) Fast fibres in a large animal: Fibre types contractile properties and myosin expression in pig skeletal muscles. The Journal of Experimantal Biology, 207, 1875-1886. doi: $10.1242 /$ jeb.00950

[21] Wada, M., Inashima, S., Yamada, T. and Matsunaga S. (2003) Endurance training-induced changes in alkali light chain patterns type IIb fibers of the rat. Journal of Applied Physiology, 94, 923-929.

[22] Wahrmann, J.P., Winand, R. and Rieu, M. (2001) Plasticity of skeletal myosin in endurance-trained rats (I): A quantitative study. European Journal of Applied Physiology, 84, 367-372. doi:10.1007/s004210100402

[23] World Medical Association Declaration of Helsinki. (1997) Recommendations guiding physicians in biomedical research involving human subjects. Cardiovascular Research, 35, 2-3. doi:10.1016/S0008-6363(97)00109-0

[24] Ledwith, A. and McGowan, C.M. (2004) Muscle biopsy: A routine diagnostic procedure. Equine Veterinary Educa- 
tion, 16, 62-67. doi:10.1111/j.2042-3292.2004.tb00268.x

[25] d'Albis, A., Pantaloni, C. and Bechet, J.J. (1979) An electrophoretic study of native myosin isozymes and of their subunit content. European Journal of Biochemistry/FEBS, 99, 261-272.

[26] Hämäläinen, N. and Pette, D. (1996) Slow-to-fast transitions in myosin expression of rat soleus muscle by phasic high-frequency stimulation. FEBS Letters, 399, 220-222. doi:10.1016/S0014-5793(96)01325-7

[27] Talmadge, R.J. and Roy, R.R. (1993) Electrophoretic separation of rat skeletal muscle myosin heavy-chain isoforms. Journal of Applied Physiology, 75, 2337-2340.

[28] Kohn, T.A. and Myburgh, K.A. (2006) Electrophoretic separation of human skeletal muscle myosin heavy chain isoforms: The importance of reducing agents. The Journal of Physiological Sciences, 56, 355-360.

[29] Oakley, B.R., Kirsch, D.R. and Morris, N.R. (1980) A simplified ultrasensitive silver stain for detecting proteins in polyacrylamide gels. Analytical Biochemistry, 105, 361363. doi:10.1016/0003-2697(80)90470-4

[30] Laemmli, U.K. (1970) Cleavage of structural proteins during the assembly of the head of bacteriophage T4. Nature, 227, 680-685.

[31] Neuhoff, V., Stamm, R. and Eibl, H. (1985) Clear background and highly sensitive protein staining with coomassie blue dyes in polyacrylamide gels: A systematic analysis. Electrophoresis, 6, 427-448.

[32] Larsson, L. and Moss, R.L. (1993) Maximum velocity of shortening in relation to myosin isoform composition in single fibres from human skeletal muscles. The Journal of Physiology, 472, 595-614.

[33] Reiser, P.J., Moss, R.L., Giulian, G.G. and Greaser, M.L.
(1985) Shortening velocity in single fibers from adult rabbit soleus muscles is correlated with myosin heavy chain composition. The Journal of Biological Chemistry, 260, 9077-9080.

[34] Leisson, K., Alev, K., Kaasik, P., Jaakma, Ü. and Seene, T. (2011) Myosin heavy chain pattern in the Akhal-Teke horses. Animal, 5, 658-662. doi: $10.1017 /$ S1751731110002375

[35] Seene, T., Alev, K., Kaasik, P., Pehme, A. and Parring, A.-M. (2005) Endurance training: Volume-dependent adaptational changes in myosin. International Journal of Sports Medicine, 26, 815-821.

[36] Seene, T., Alev, K., Kaasik, P. and Pehme, A. (2007) Changes in fast-twitch muscle oxidative capacity and myosin isoforms modulation during endurance training. Journal of Sports Medicine and Physical Fitness, 47, 124-132.

[37] Bottinelli, R. (2001) Functional heterogeneity of mammalian single muscle fibres: Do myosin isoforms tell the whole story? Pflügers Archiv: European Journal of Physiology, 443, 6-17.

[38] Eimre, M., Puhke, R., Alev, K., Seppet, E., Sikkut, A., Peet, N., Kadaja, L., Lenzner, A., Haviko, T., Seene, T., Saks, V.A. and Seppet, E.K. (2006) Altered mitochondrial apparent affinity for ADP and impaired function of mitochondrial creatine kinase in Gluteus medius of patients with hip osteoarthritis. American Journal of PhysiologyRegulatory, Integrative and Comparative Physiology, 290, R1271-R1275. doi:10.1152/ajpregu.00651.2005

[39] Neunhäuserer, D., Zebedin, M., Obermoser, M., Moser, G., Tauber, M., Niebauer, J., Resch, H. and Galler, S. (2011) Human skeletal muscle: Transition between fast and slow fibre types. Pflügers Archiv: European Journal of Physiology, 461, 537-543. 